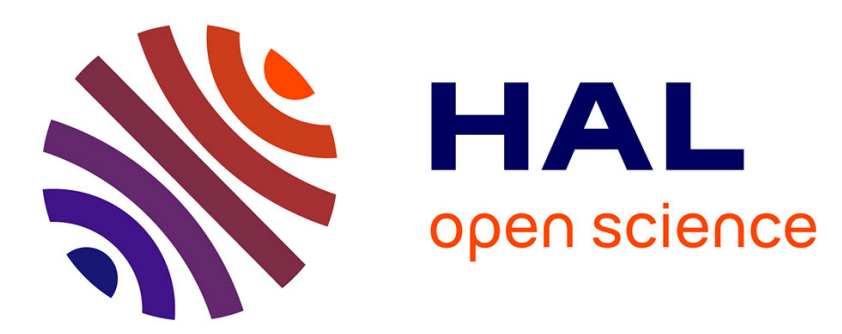

\title{
Ultrasound velocimetry in a shear-thickening wormlike micellar solution: Evidence for the coexistence of radial and vorticity shear bands
}

Vishweshwara Herle, Sébastien Manneville, Peter Fischer

\section{To cite this version:}

Vishweshwara Herle, Sébastien Manneville, Peter Fischer. Ultrasound velocimetry in a shearthickening wormlike micellar solution: Evidence for the coexistence of radial and vorticity shear bands. European Physical Journal E: Soft matter and biological physics, 2008, 26 (1-2), pp.3-12. 10.1140/epje/i2007-10304-3 . ensl-00526262

\section{HAL Id: ensl-00526262 \\ https://hal-ens-lyon.archives-ouvertes.fr/ensl-00526262}

Submitted on 14 Oct 2010

HAL is a multi-disciplinary open access archive for the deposit and dissemination of scientific research documents, whether they are published or not. The documents may come from teaching and research institutions in France or abroad, or from public or private research centers.
L'archive ouverte pluridisciplinaire HAL, est destinée au dépôt et à la diffusion de documents scientifiques de niveau recherche, publiés ou non, émanant des établissements d'enseignement et de recherche français ou étrangers, des laboratoires publics ou privés. 


\title{
Ultrasound velocimetry in a shear-thickening wormlike micellar solution: Evidence for the coexistance of radial and vorticity shear-bands
}

\author{
Vishweshwara Herle ${ }^{1 \mathrm{a}}$, Sebastien Manneville ${ }^{2}$, and Peter Fischer ${ }^{1}$ \\ 1 Institute of Food Science and Nutrition, ETH Zurich, 8092 Zurich, Switzerland \\ ${ }^{2}$ Ecole Normale Supérieure de Lyon, Laboratoire de Physique, 46 allée d'Italie, 69364 Lyon cedex 07, France
}

Received: date / Revised version: date

\begin{abstract}
Pointwise local velocity measurements carried out on $40 \mathrm{mM}$ cetylpyridinium chloride - sodium salicylate (CPyCl-NaSal) wormlike solution using high frequency ultrasound velocimetry in a Couette shear cell is reported. The studied wormlike solution exhibits Newtonian, shear-thinning and shear-thickening rheological behavior in a stress controlled environment. Previous rheology, flow visualization and small angle light/neutron scattering experiments in the shear-thickening regime of this system showed the presence of stress driven alternating transparent and turbid rings or vorticity bands along the axis of the Couette geometry. In contrast to other wormlike micellar systems, local velocity measurements reveal homogenous flow inside the $1 \mathrm{~mm}$ gap of the Couette cell in the shear-thinning (stress-plateau) region. Only when the solution is sheared beyond the critical shear stress (shear-thickening regime) in stress-controlled experiment, we observe inhomogenous flow characterized by radial or velocity gradient shear-bands with a highly sheared band near the rotor and a weakly sheared band near the stator of the Couette geometry. Furthermore, fast measurements performed in the shear-thickening regime to capture the temporal evolution of local velocities, indicate coexistance of both radial and vorticity shear-bands. However the same measurements carried out in shear rate controlled mode of the rheometer do not show such rhelogical complexity.
\end{abstract}

PACS. 83.80.Qr Surfactant and micellar systems, associated polymers - 83.60.Wc Flow Instabilities 47.50.-d Non-Newtonian fluid flows $-43.58 .+\mathrm{z}$ Acoustical measurements and instrumentation

\footnotetext{
a Present address: Department of Physics, University of Fribourg, CH-1700, Fribourg, Switzerland
} 


\section{Introduction}

Addition of small amounts of counter-ions or co-surfactants to certain cationic surfactants leads to the formation of long threadlike or wormlike micelles [1]. Aqueous solutions of these wormlike micelles are viscoelastic and their rheological behavior has been widely studied during the past 20 years by several authors using many different experimental techniques [1-8]. The unique property of breakage and recombination of wormlike micelles and the large length scales in these systems induces a wide variety of complex structures under shear flow resulting in shear induced structures (SIS) [9-15]. The formation of SIS in most cases is associated with shear bands, i.e. the formation of spatially inhomogeneous regimes. This leads to different stress or strain levels in the sheared solution and is expressed theoretically by a multibranched constitutive equation [16-24]. In most cases the shear bands develop in the velocity gradient (or radial) direction in Couette flow. However shear bands in the neutral (or vorticity) direction have also been observed for semidilute shear-thickening wormlike micellar solutions and are termed as vorticity bands $[5,6,13,25,26]$. Furthermore, both type of shear bands are usually transient in nature: in velocity gradient bands, the boundary between the bands moves radially and the vorticity bands undergo a flip-flop like change axially. This spatio-transient behavior of shear bands with different structures and inturn different viscosities, manifests itself in oscillations of the overall rheological signals or rheochaotic behavior [8,13,27-30]. While many of the theoretical work addresses the formation of radial or velocity gradient shear bands $[18,29,31]$, only a few attempts have been made towards explaining the vorticity bands $[32-34]$.

In this article we focus on an equimolar $(40 \mathrm{mM})$ mixture of cetylpyridinium chloride and sodium salicylate, a well known surfactant-salt system that forms wormlike micelles in aqueous media $[5,6]$. Under linear flow conditions this particular solution behaves as a Maxwellian fluid with a single relaxation time, $\lambda=0.02 \mathrm{~s}$, and a plateau modulus, $\mathrm{G}_{0}$ $=6.3 \mathrm{~Pa}$. However, the rheological response is very complex in the non-linear regime. At low shear stresses the solution initially shows Newtonian behavior followed by shear-thinning properties. At stresses beyond a critical stress, $\tau_{c}$, the solution exhibits shear-thickening behavior. These rheological transitions are accompanied by the following optical behavior: (i) at rest and in the Newtonian flow region the sample is optically transparent, (ii) in the shear-thinning regime the entire solution turns slightly turbid, and (iii) in the subsequent shear-thickening regime transparent and turbid rings suddenly appear in the vorticity (neutral) direction of the rheometer geometry [13,25,26]. These vorticity bands are observed in all standard rheometrical geometries, i.e., in Couette, cone-plate and in parallel plate systems. In cone-plate and parallel plate geometries the bands appear as concentric rings [6,25] and in Couette geometry, they are stacked one above the other along the cylinder length [13,26]. A unique behaviour of these vorticity bands is that they alternate between their transparent and turbid states in a regular fashion [25,26]. This leads to periodic oscillations in the shear rate $(\dot{\gamma})$, and apparent viscosity $\eta$, in stress-controlled experiments [25]. As long as the shear stress is kept constant $\left(\tau \geqslant \tau_{c}\right)$ the bands continue to alternate and the material never reaches a stationary state [5, $6,13,25,26]$. Rheological, flow visualization and rheo small angle light scattering studies of this system have indicated that these bands are stress-driven and the frequency of oscillations in $\dot{\gamma}$ and $\eta$ are correlated to the frequency of appearance and disappearance of the bands, which is approximately $2 \mathrm{~Hz}$. From rheo small angle neutron scattering 
(SANS) measurements in the Newtonian and shear-thinning flow region it was shown that there was only a minor alignment of the wormlike micelles in the flow direction [26]. The anisotropic SANS intensity maps obtained from the triggered Rheo-SANS experiments and the anisotropy factor $\left(A_{f}\right)$ calculated from the patterns indicated that both the oscillating transparent and turbid bands consist of highly anisotropic structures as compared to the structures in the shear-thinning regime with the turbid shear-thickening vorticity band having the highest anisotropy. Furthermore, by high-speed video imaging experiments we have also shown that the turbid band exhibits a high viscous flow response and that a phase shift $(\simeq \pi / 2)$ between the structural build up of the turbid band and the rheological response function forces the sample in the continuous oscillation of the free rheological parameter $(f \simeq 2 \mathrm{~Hz})[26]$.

One of the aims of the present work is to verify the findings of the video imaging experiments by measuring the local viscosities in each vorticity band using ultrasound velocity profiling (UVP). The UVP technique is based on timedomain cross-correlation of high-frequency ultrasonic signals backscattered by tracer particles seeded in the flowing solution [35]. UVP is a noninvasive velocimetric technique to measure local viscosities at microscopic resolutions inside the gap of rheometric geometry. Figure 1a shows the typical linear velocity profile obtained by the UVP method for a Newtonian fluid being sheared in the gap of a Couette geometry. In this case the inner cylinder with radius $\mathrm{R}_{i}$ is rotating while the outer cylinder of radius $\mathrm{R}_{o}$ is stationary. Hence the velocity is maximum at $\mathrm{R}_{i}$ and zero at $\mathrm{R}_{o}$. However the situation is completely different for a solution forming radial shear bands in the gap. During such a shear banding flow condition, the sheared fluid separates into two differently sheared regions and consequently, in a Couette gometry, one observes a highly sheared band near the rotating cylinder $\left(\mathrm{R}_{i}\right)$ and a weakly sheared band at the stationary wall $\left(\mathrm{R}_{o}\right)$. Figure $1 \mathrm{~b}$ shows a shear-banded velocity profile with the boundary of the band located at a distance $\delta$ from the rotating wall. Furthermore, if such a radial shear band is transient in nature, then this boundary will move within the gap of the Couette geometry as indicated by the horizontal arrow in Fig. 1b. The system studied here, that is, $40 \mathrm{mM}$ CPyCl-NaSal solution, shows vorticity bands which oscillate in the neutral direction. In that situation we expect the velocity profile to be similar to Fig. 1c, with one of the bands having different local viscosity than the other and teh bands moving axially as indicated by the vertical arrow. Furthermore if a situation arises wherein one observes the co-exsitance of both (radial and vorticity) types of shear-bands and if both show spatio-temporal oscillations then velocity profile for such a situation would resemble Fig. 1d.

Even though extensive investigations have been carried out to analyze the microstructures and the resulting viscosities in the vorticity bands formed in a solution of $40 \mathrm{mM} \mathrm{CPyCl-NaSal}$, the system was never studied for the presence or absence of radial or velocity gradient bands. Our goal here is to identify the type of shear bands and to obtain an idea about the local dynamics within each band. We perform the local velocity measurements in the $1 \mathrm{~mm}$ gap of the Couette geometry using the UVP method. Experiments in both controlled shear stress and shear rate conditions are described and discussed. The pointwise local velocity measurements are used to identify the flow inside the gap of the Couette cell. In the shear-thickening regime, we observe radial shear bands with a high shear rate band $\left(\dot{\gamma}_{1}\right)$ at the rotating wall and a low shear rate band $\left(\dot{\gamma}_{2}\right)$ at the stationary wall of the Couette shear cell. With the help of very rapid data aquisition, fast UVP measurements were performed to capture the temporal behavior of the local shear rates. Analyzing the oscillating shear rates in each radial band and comparing the frequency of oscillation to 

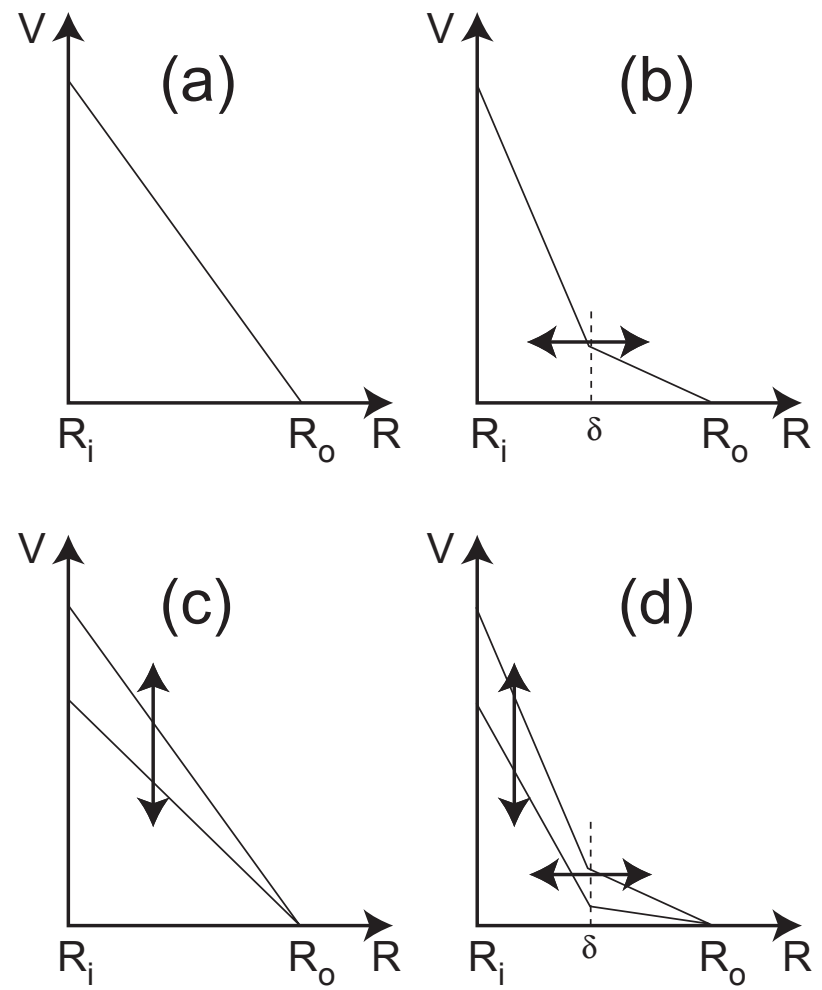

Fig. 1. Velocity profiles for (a) Newtonian fluid; (b) fluid showing radial shear-bands; (c) fluid with vorticity banding; and (d) fluid showing both the radial and vortity bands. $\mathrm{R}_{i}$ and $\mathrm{R}_{o}$ corresponds to the radii of inner (rotating) and outer (stationary) cylinders of the Couette cell while $\delta$ is the boundary between the radial shear bands. The arrows indicate temporal fluctuations in the shear bands.

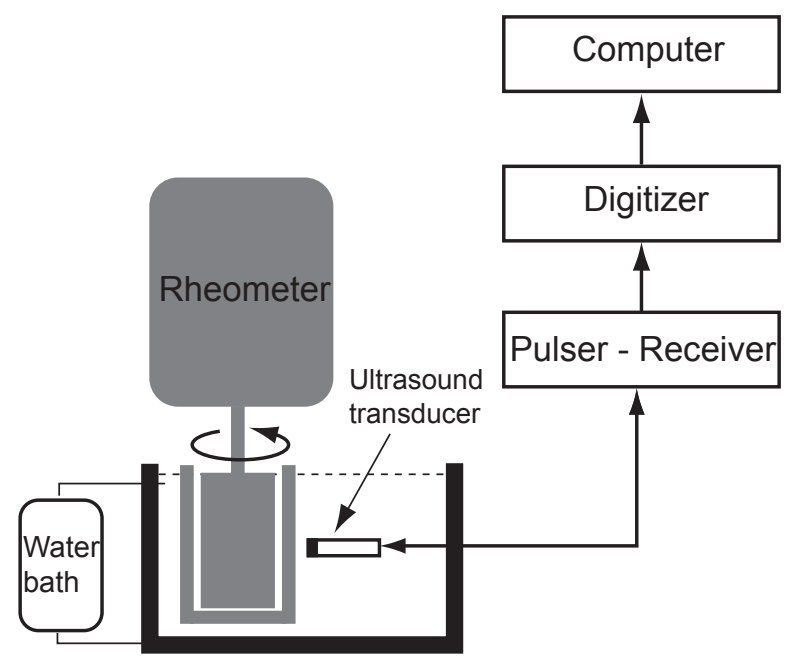

Fig. 2. Ultrasound velocity profiling (UVP) experimental set-up. Figure adapted from Manneville et al. [35]

that of the flip-flop behavior of vorticity bands we show that there is a coexistence of both radial and vorticity bands in this system.

The manuscript is organized as follows: In Section-2 the materials and experimental setting are discussed briefly. Results for both stress controlled and strain controlled rheo-UVP experiments are presented and discussed in Section-3 before presenting a summary of the investigations in the final Section. 


\section{Experimental}

\subsection{Materials}

Cetylpyridiunum chloride $(\mathrm{CPyCl})$ and sodium salicylate (NaSal) were obtained from Fluka (Buchs, Switzerland) and used without further purification. A solution of CPyCl-NaSal in water is usally transparent to ultrasonic waves. Thus, in order to measure the velocity profiles, $1 \mathrm{wt} \%$ hollow glass spheres (Sphericel, Potters Industries) were added as tracer particles. Required amount of $\mathrm{CPyCl}$ to prepare a $40 \mathrm{mM}$ solution was first weighed and dissolved in Milipore water. Then, the glass spheres of mean diameter $11 \mu \mathrm{m}$ were added to this solution. Once these spheres are homogeneously dispersed in water, NaSal was slowly added. This helps in slow build-up of viscosity in the sample due to the growth of wormlike micelles in the presence of dispersed glass spheres.

\subsection{UVP - Experimental setup}

Simultaneous rheology and local velocity data are measured in a stress-controlled rheometer (TA Instruments AR 1000), equipped with a transparent Couette geometry. The Couette cell was made of smooth Plexiglas with inner cylinder radius of $24 \mathrm{~mm}$, gap $1 \mathrm{~mm}$ and height $30 \mathrm{~mm}$. This whole cell is surrounded by circulated water whose temperature was kept constant at $21^{\circ} \mathrm{C}$. Figure 2 shows a schematic of the UVP experimental set-up. A PVDF piezo-polymer transducer (Panametrics PI 50-2) immersed in the water in front of the stator generates focussed ultrasonic pulses of central frequency $f=36 \mathrm{MHz}$. The transducer is controlled by a pulser-receiver unit (Panametrics 5900PR). Ultrasonic pulses travel through the $1 \mathrm{~mm}$ gap of the Couette geometry and are scattered by glass spheres. Backscattered signals are then collected and stored on a high-speed PCI digitizer (Acqiris DP235), and transfered to the host computer for post processing. The spatial resolution of the experimental setup is of the order of $40 \mu \mathrm{m}$ and the temporal resolution ranges between 0.02 and $2 \mathrm{~s}$ per profile. The experimental setup is shown in Fig. 2. Details of the experimental set-up, calibration procedure, and data analysis are described in detail by Manneville et.al [35].

\section{Results and Discussion}

\subsection{Stress-controlled rheology}

Figure 3a displays the flow curve of the surfactant solution seeded with $1 \mathrm{wt} \%$ glass spheres. The overall nature of the flow curve did not change with addition of the particles, however the critical stress, $\tau_{c}$, where the solution exhibits shear-thickening behavior is found to be $10 \mathrm{~Pa}$ as compared to $13 \mathrm{~Pa}$ observed for a tracer particle free solution. As described previously, when increasing the shear stress, the solution exhibits Newtonian, shear-thinning and shearthickening behaviors $[5,25,26]$. For reasons of clarity, the shear-thickening regime is not shown in the main figure, but the inset shows entire flow curve including the shear-thickening regime. The viscosity drops drastically between 8 and $10 \mathrm{~Pa}$ and it is between these shear stresses that a quasi-horizontal stress-plateau is observed (Fig. 3a). In comparison to the unseeded solution (see [25]) the shear thinning plateau is covering a wider range of shear rates. Such a plateau 
(a)

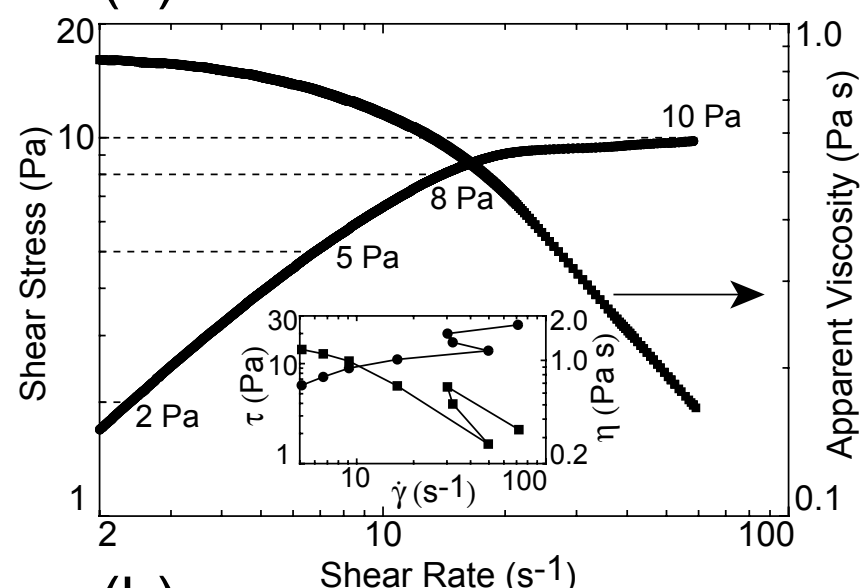

(b)

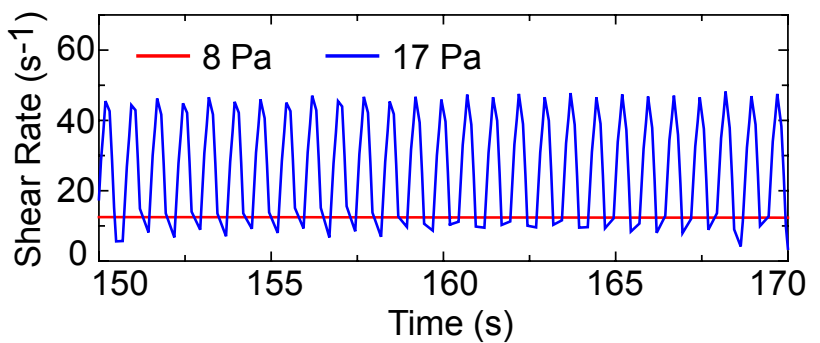

Fig. 3. Color online: (a) Flow curve of $40 \mathrm{mM}$ CPyCl-NaSal solution $(1<\tau<10 \mathrm{~Pa})$ with 1 wt\% tracer particles recorded under controlled shear stress conditions. The inset shows the full flow curve including the shear thickening region. Horizontal dotted lines represent the stresses where the steady state UVP velocity profiles were recorded. (b) Transient shear rate signals below $(8 \mathrm{~Pa})$ and above $(17 \mathrm{~Pa})$ shear-thickening regime of flow.

in $\tau$ vs $\dot{\gamma}$ has been accounted for shear-induced transitions or formation of shear-induced structures (SIS) [1,36-39] and studied in the framework for shear banding theories $[18,40,41]$. Further shearing the sample above the critical shear stress $\left(\tau_{c}=10 \mathrm{~Pa}\right)$, the solution exhibits shear-thickening behavior (inset in Fig. 3a) and this region extends up to $\tau=17 \mathrm{~Pa}$ as compared to $25 \mathrm{~Pa}$ in the case of the unseeded sample. In this regime of flow, strong temporal oscillations in apparent viscosity and shear rate as well as the flip-flop like axial motion of the vorticity bands has previously been observed which correspond to fast structural changes in the system $[5,25,26]$. Figure $3 \mathrm{~b}$, shows the variation of shear rate as a function of time at 8 and $17 \mathrm{~Pa}$. The shear rate is constant for $8 \mathrm{~Pa}$ which is below the shear-thickening regime. However in the shear thickening regime that follows (i.e., at $17 \mathrm{~Pa}$ ), the shear rate signal shows large temporal oscillations around a mean value with a characteristic frequency. To investigate the structural transitions in these regimes of flow (shear-thinning and shear-thickening) and to get an idea about the local velocities in shear bands, pointwise local velocity measurements are carried out inside the gap of a Couette cell.

\subsection{Rheology and UVP measurements $(\tau \leqslant 10 \mathrm{~Pa})$}

To begin with, several shear stresses below $\tau_{c}=10 \mathrm{~Pa}$ are chosen to measure the velocity profiles (dotted lines in Fig. 3). Figure 4a shows the rheological protocol used to measure such profiles. At $t=0$, a shear stress of $\tau$ is instantly 


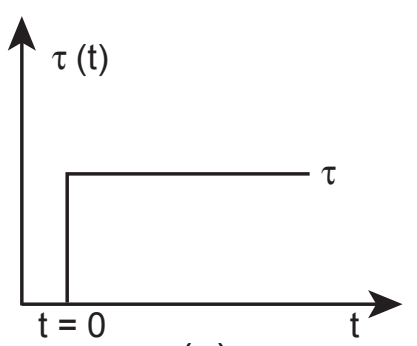

(a)

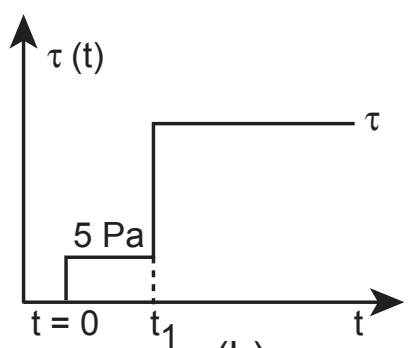

(b)

Fig. 4. Rheological test sequence used to measure velocity profiles. (a) Step stress method for shear stresses $\tau \leqslant 10 \mathrm{~Pa}$ and (b) Pre-shear and step stress method for shear stresses $\tau>10 \mathrm{~Pa} ; \mathrm{t}_{1}=40 \mathrm{~s}$.
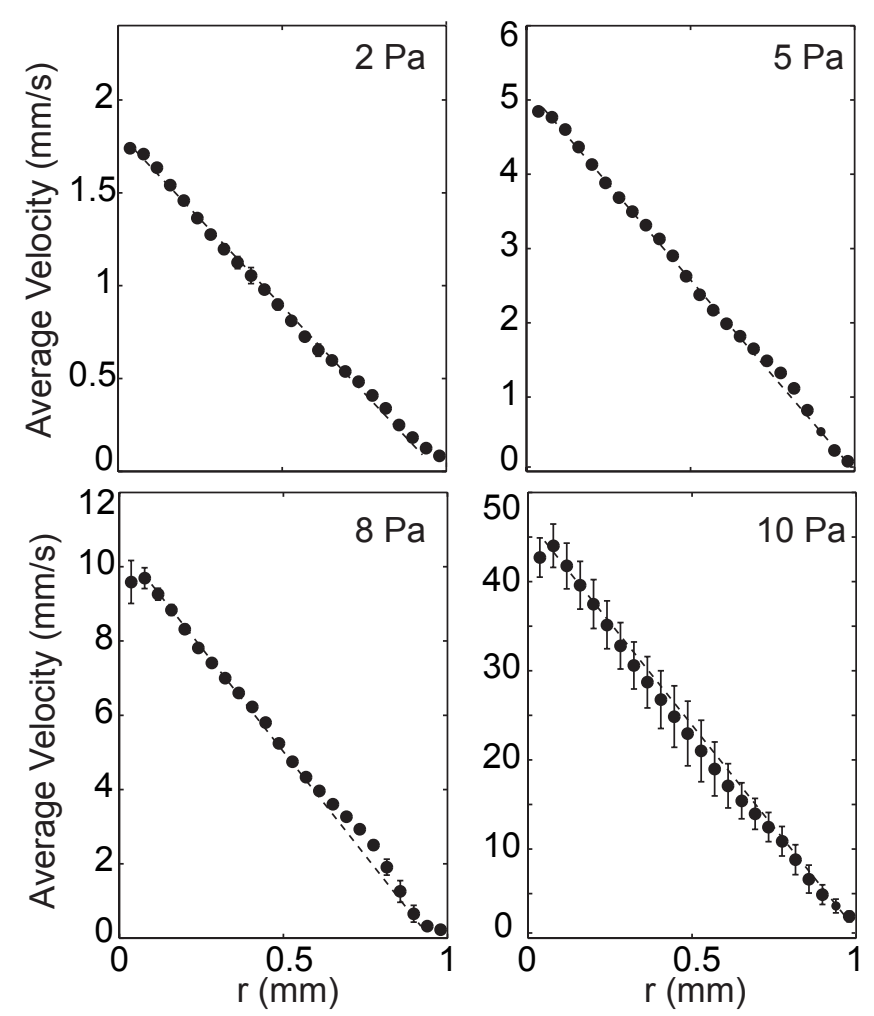

Fig. 5. Time-averaged velocity profiles for different shear stresses in the Newtonian and shear-thinning regime measured in the stress-controlled mode. Error bars correspond to the standard deviation of the local velocities and mainly account for temporal fluctuations in the velocity. $r$ denotes the radial distance to the rotor; $r=0$ at the rotor and $r=1$ at the stator.

applied and the velocity profiles are recorded for a minimum of $200 \mathrm{~s}$. These are then averaged and such time-averaged velocity profiles are shown in Fig. 5 for different shear stresses. $r$ denotes the radial distance in the gap of the Couette shear cell $(r=0$ at the rotor and $r=1$ at the stator). For $\tau=2 \mathrm{~Pa}$, the profile is very close to a straight line, consistent with the Newtonian behavior of the micellar solution at low shear stresses. However, for $\tau=10$, the data do not exactly fall on the Newtonian velocity profile but rather deviates significantly. Such a curvature in the velocity profile is due to the sharp shear-thinning effect observed at $\tau=10$ but the overall flow behavior of the solution in $1 \mathrm{~mm}$ gap of the shear cell is homogenuos. Furthermore, temporal fluctuations of velocity come in to play at this shear stress. These temporal fluctuations in the measured velocity profiles are due to oscillations in apparent viscosity which start to appear at this critical shear stress $\left(\tau_{c}=10 \mathrm{~Pa}\right)$. 

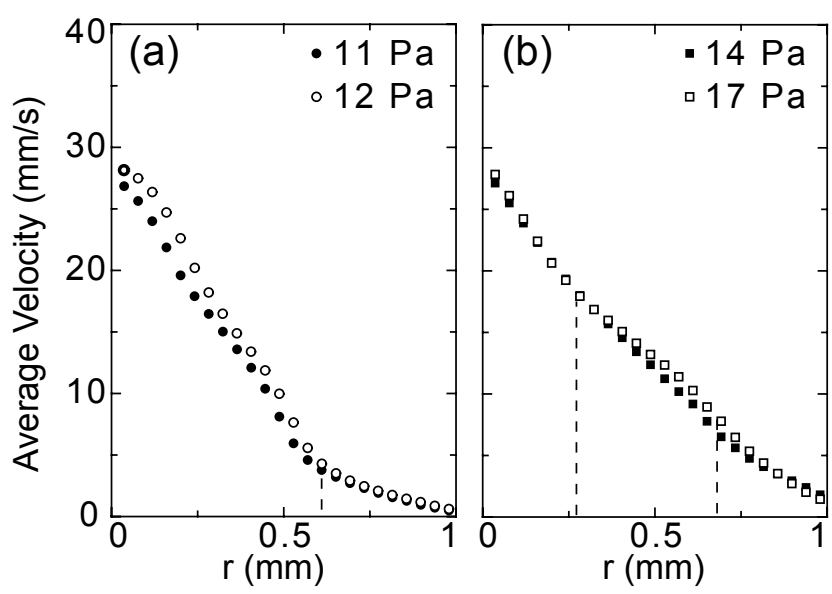

Fig. 6. Time-averaged velocity profiles for different shear stresses in the shear-thickening regime measured in the stress-controlled mode. $r$ denotes the radial distance to the rotor; $r=0$ at the rotor and $r=1$ at the stator. Dotted vertical lines indicate position of the interface between the radial bands.

In most of the experimental works dealing with surfactant systems, it has been argued that a strong flow-structure coupling induces new organizations like nematic phases or onion textures or other flow-induced phenomena [14,4245]. With the evolution of these new structures, the systems exhibit a stress-plateau in steady-state flow curve $(\tau$ vs $\dot{\gamma}$ ), which in the case of present seeded material would be $\tau_{c}=10 \mathrm{~Pa}$. Under such flow conditions it is believed that the sheared fluid separates into two differently sheared regions and such a behavior is termed as shear-banding flow. In a Couette shear cell under shear-banding situation, one observes a highly sheared band near the rotating cylinder and a weakly sheared band at the stationary wall. Those systems exhibiting stress-plateau behavior have been investigated by ultrasound velocimetry [23,24,46], flow-birefringence [39], small angle neutron scattering [47], particle image velocimetry (PIV) [48,49] and NMR velocity measurements [50], and an inhomogeneous flow in the gap of the Couette cell has indeed been observed with the presence of high and low shear bands. Since such an inhomogeneous flow behavior has been experimentally observed in many surfactant systems, the presence of a stressplateau is considered as the hallmark of shear-banding flows (See Fig. 1b). This generality has motivated many recent theoretical works involving phenomenological models as well as microscopic approaches [29,51-53]. In our case, rheologically, a quasi-horizontal stress plateau is indeed observed between $\tau=8$ and $10 \mathrm{~Pa}$. However, in contrast to the other works mentioned above, this solution does not show any inhomogeneous flow in the plateau region. This is a surprising finding and thus questions the universal feature of existence of shear bands in the stress-plateau region.

\subsection{Transient UVP measurements $(\tau>10 \mathrm{~Pa})$}

\section{(a) Time-averaged velocity profiles}

As shown in the inset of Fig. 3, the solution shows shear-thickening behavior above $10 \mathrm{~Pa}$. Previous study of the system showed formation of alternating vorticity bands in this flow regime with oscillations in the apparent viscosity and shear rate $[25,26]$. Hence one expects a rich variety of time-dependent phenomena, such as large temporal oscillations or rheochaotic behavior of the velocity in the shear-thickening regime. To investigate these fast time-dependent behaviors, 
careful rheological protocols should be used while measuring the velocity in the annular gap. Figure $4 \mathrm{~b}$, shows such an experimental protocol used to measure transient velocity profiles. A step stress of $5 \mathrm{~Pa}$ is applied at time $\mathrm{t}=0$ and the solution is pre-sheared for $40 \mathrm{~s}$ at this shear stress. Then the sample is subjected to a higher stress $\left(\tau>\tau_{c}\right)$ for more than $1000 \mathrm{~s}$ and in this time period, transient velocity profiles are recorded at a rate of 20 profiles per second. Such very fast measurements help us to capture fast dynamics in this system.

Figure 6 shows time-averaged velocity profiles for different shear stresses in the shear-thickening region. Typically more than 200 profiles have been averaged to obtain this data. Measurements above $17 \mathrm{~Pa}$ could not be performed due to stronger viscoelastic effects (rod-climbing). Figure 6a shows the average velocity profiles measured for 11 and $12 \mathrm{~Pa}$ close to $\tau_{c}$ and Fig. $6 \mathrm{~b}$ shows the same for 14 and $17 \mathrm{~Pa}$ further away from $\tau_{c}$. All four profiles show large temporal fluctuations which might correspond to the flip-flop like motion of the vorticity bands. The standard deviation is not shown in this figure for reasons of clarity but it will be discussed in the next section. Compared to the velocity profiles of Fig. $5\left(\tau \leqslant \tau_{c}\right)$, these profiles clearly reveal an inhomogeneous flow with an unambiguous radial banding structure. For shear stresses close to $\tau_{c}$ (Fig. 6a), two differently sheared bands coexist in $1 \mathrm{~mm}$ gap of the Couette geometry with the interface of the bands located approximately at $r \simeq 0.6 \mathrm{~mm}$. This is in accordance to the classical picture of shear-banding flow, with a highly sheared band located at the rotor accompanied by a low shear band near the stator $[8,23,39,44,45,50,54]$. This is the first report on radial banding in the shear-thickening $40 \mathrm{mM}$ CPyCl-NaSal wormlike micellar solution which is a very surprising and new phenomenon observed for this system. Until now only vorticity bands were studied for this system $\left(\tau \geqslant \tau_{c}\right)$. Here, local velocimetry clearly shows that the flow behavior of this solution is more complex in the shear-thickening regime.

Velocity profiles in Fig. 6a presents only a single interface whereas three differently sheared regions (different slopes in the velocity profile) can be seen for shear stresses $\tau=14$ and $17 \mathrm{~Pa}$ as displayed in Fig. 6b. This indicates the presence of three shear bands in the annular gap. Furthermore, at these stresses the flow becomes very complex with large fluctuations in the velocity. These temporal fluctuations will be discussed in the next section. We have to mention here that the three banded situation appears only momentarily for $\tau=14 \mathrm{~Pa}$ but the third band becomes more pronounced with increase in the shear stress $(\tau=17 \mathrm{~Pa})$. The average position of the interface for low shear band almost remains same $(\delta \simeq 0.6 \mathrm{~mm})$, however, the highly sheared band splits into two bands with the new interface being at $\delta \simeq 0.25 \mathrm{~mm}$. We have to remind the reader that the position of the interface is an average value and it is very interesting to see that when increasing the shear stress from 11 to $17 \mathrm{~Pa}$, the average position of the interface, $\delta$, does not move towards the stator as observed by other authors $[8,40,44,45]$. However, development of the radial bands is as described in the literature, which is by nucleation and growth mechanism. Indeed, start up measurements between $\mathrm{t}_{1}<\mathrm{t}<\mathrm{t}_{1}+10 \mathrm{~s}$ (data not shown) showed that a high shear band nucleates at the inner wall and grows rapidly towards the stator before stabilizing approximately at $\delta \simeq 0.6 \mathrm{~mm}$. This mechanism of nucleation and growth is very rapid as compared to other systems wherein it can take few minutes to achieve a stable interface. In all cases $(\tau=11$ to $17 \mathrm{~Pa})$, the flow presents large fluctuations of the velocity in each band with oscillating interface and even in the time domain $\mathrm{t}_{1}<\mathrm{t}<\mathrm{t}_{1}+10 \mathrm{~s}$, the average position of the interface fluctuates continuously. 


\section{(b) Temporal evolution of local shear rates and interface dynamics}

As mentioned above, large temporal fluctuations in the local velocity are observed in each band. Assuming no wall slip, if $v_{1}(t)$ denotes the rotor velocity, $\delta(t)$, the position of the interface, and $v(\delta(t), t)$, the velocity at the interface, then the local shear rates in the weakly sheared band, $\dot{\gamma}_{2}$, and in the high shear band, $\dot{\gamma}_{1}$, can be calculated using the following equations $[45]$ :

$$
\begin{gathered}
\dot{\gamma}_{2}=\frac{\left(R_{1}+\delta\right)^{2}+R_{2}^{2}}{\left(R_{1}+\delta\right)\left(R_{1}+R_{2}+\delta\right)} \cdot \frac{v(\delta)}{(e-\delta)} \\
\dot{\gamma}_{1}=\frac{\left(R_{1}+\delta\right)^{2}+R_{1}^{2}}{R_{1}\left(2 R_{1}+\delta\right)} \cdot \frac{v_{1}-\frac{R_{1}}{R_{1}+\delta} \cdot v(\delta)}{\delta}
\end{gathered}
$$

where $R_{1}$ and $R_{2}$ are the inner and outer radii of the Couette geometry and $e=\left(R_{2}-R_{1}\right)$.

Figure 7 shows the evolution of local shear rates and the position of the interface as a function of time. Because of the fluctuations in the local velocities, one observes oscillations in the local shear rates $\left(\dot{\gamma}_{1}, \dot{\gamma}_{2}\right)$ in each band. A closer look at these oscillations show that $\dot{\gamma}_{1}$ and $\dot{\gamma}_{2}$ are anti-correlated but $\delta(t)$ seems to be in phase with $\dot{\gamma}_{2}$ (at-least better correlated than to $\dot{\gamma}_{1}$ ). The position of the interface between the two bands also oscillates around a mean value of $\delta \simeq 0.6 \mathrm{~mm}$. Figure 8 displays the similar results for $\tau=14 \mathrm{~Pa}$. Here we show the temporal oscillations in only two bands as the third band was appearing at irregular intervals. The oscillations in $\dot{\gamma}_{1}$ and $\dot{\gamma}_{2}$ for $\tau=14$ Pa, are not as regular as for $\tau=11$ and $12 \mathrm{~Pa}$ (Fig. 7) and also the amplitude of the oscillations in $\delta(t)$ for $\tau=14 \mathrm{~Pa}$ is larger than in Fig. 7 ( $\tau=11,12 \mathrm{~Pa})$. Furthermore, in the case of $\tau=14 \mathrm{~Pa}$, the relative fluctuations of interface position is about $22 \%$ as compared to $10 \%$ for 11 and $12 \mathrm{~Pa}$. It is quite clear that increase in shear stress causes larger fluctuations in the local velocity and also in the interface position which is a sign of more chaotic like flow behavior. This point is further supported in Fig. 9a, which shows the local shear rates for $\tau=17$ Pa. Here, instead of two bands, we clearly see a three-band situation and it becomes difficult to figure out the exact positions of the interface. Figure $9 \mathrm{~b}$ shows the regions of velocity profiles which are used to calculate three local shear rates $\left(\dot{\gamma}_{1}, \dot{\gamma}_{2}\right.$, and $\left.\dot{\gamma}_{3}\right)$. Similar to Fig. 7 , $(\tau=11,12 \mathrm{~Pa})$ the highly sheared band, $\dot{\gamma}_{1}$, and weakly sheared band, $\dot{\gamma}_{3}$, are anti-correlated. However there is no obvious correlation between the third band in the middle and two other bands. At certain time intervals the local shear rates in all the three bands are equal and the flow seems to be homogenous (see for $178 \mathrm{~s}$ in Fig. 9a). However, such an homogenous flow is very short lived (not more than $1 \mathrm{~s}$ ) and three-band scenario appears again.

As discussed in Fig. 1 we expected to observe a clear difference in the velocity profiles of the alternating transparent and turbid bands. However, the Rheo-UVP experiments indicate an additional feature of radial banding and no clear identification of the vorticity bands. The flip-flop like axial motion of the vorticity bands seems to be hidden in the observed temporal fluctuations of the UVP profiles. This means that the difference in the viscosity of both bands is hidden in the noise and the radial banding. Assuming the presence of both radial and vorticity banding one would expect to observe a velocity profile as depicted in Fig 1d. Here the different slopes of the velocity profiles and consequently the different values of the viscosities would fluctuate not only in the magnitude of the velocity but also in 

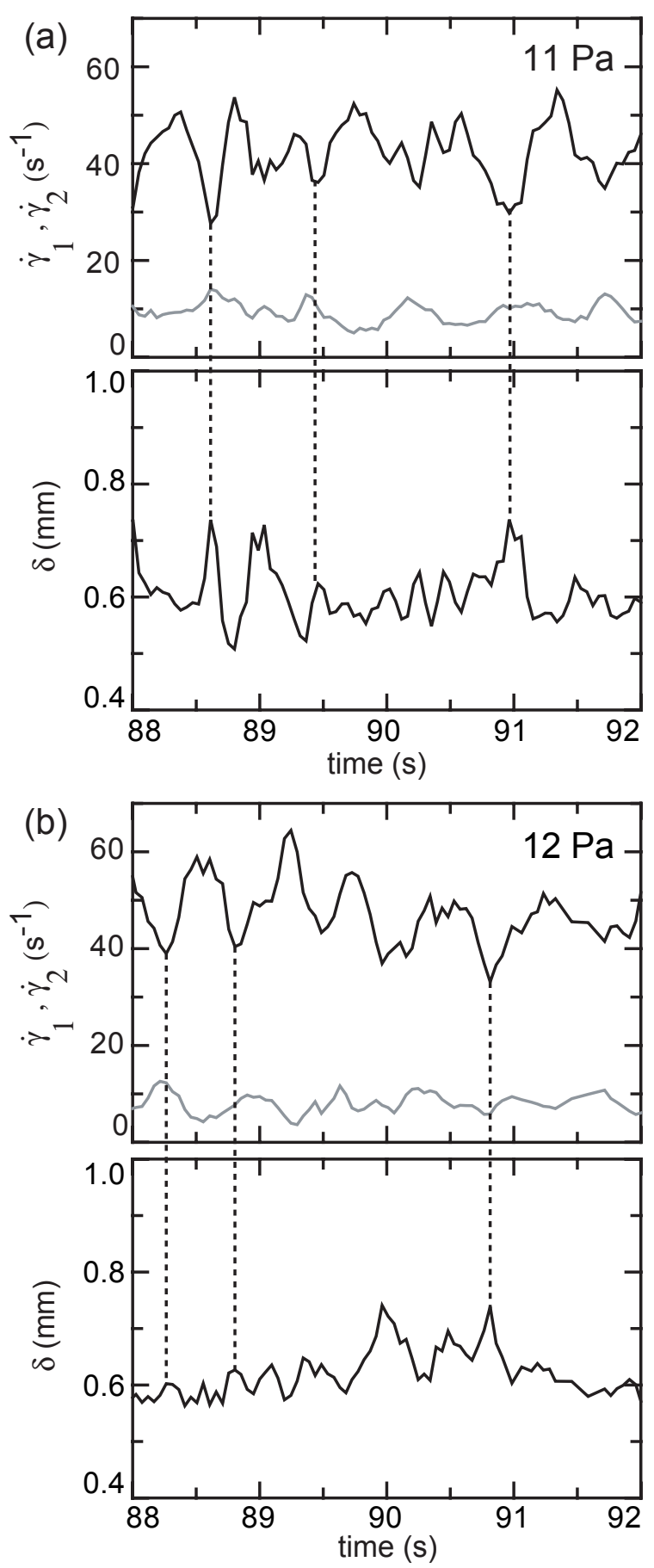

Fig. 7. Local shear rates $\left(\dot{\gamma}_{1}, \dot{\gamma}_{2}\right)$ and position of the interface $(\delta)$ as a function of time for (a) 11 Pa and (b) 12 Pa. The black and the gray lines on the top section of each figure correspond respectively to the highly sheared band $\left(\dot{\gamma}_{1}\right)$ near the rotor and to the weakly sheared band $\left(\dot{\gamma}_{2}\right)$ near the stator.

the position of the boundary. Since the velocity at the rotating cylinder does not change $(\Delta v \approx 0)$, a velocity difference resulting in the different viscosities must be generated by different slope of the individual radial bands of each vorticity band. Due to the addition of glass spheres (seeding for UVP), the whole sample becomes turbid (milky) and hence a simultaneous visual observation of transparent or turbid bands with the UVP experiments was not possible. Therefore alternative measures have to be taken to access the information about the presence or absence of vorticity bands. This 


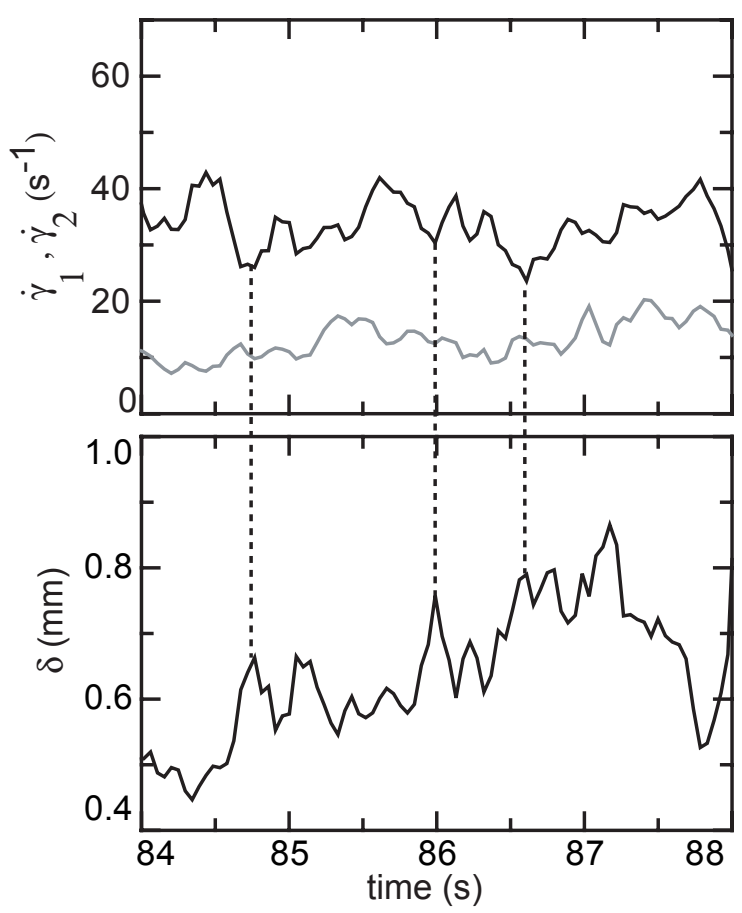

Fig. 8. Local shear rates $\left(\dot{\gamma}_{1}, \dot{\gamma}_{2}\right)$ and position of the interface $(\delta)$ as a function of time for a shear stress of 14 Pa. The black and the gray lines on the top part of the figure correspond respectively to the highly sheared band $\left(\dot{\gamma}_{1}\right)$ near the rotor and to the weakly sheared band $\left(\dot{\gamma}_{2}\right)$ near the stator.
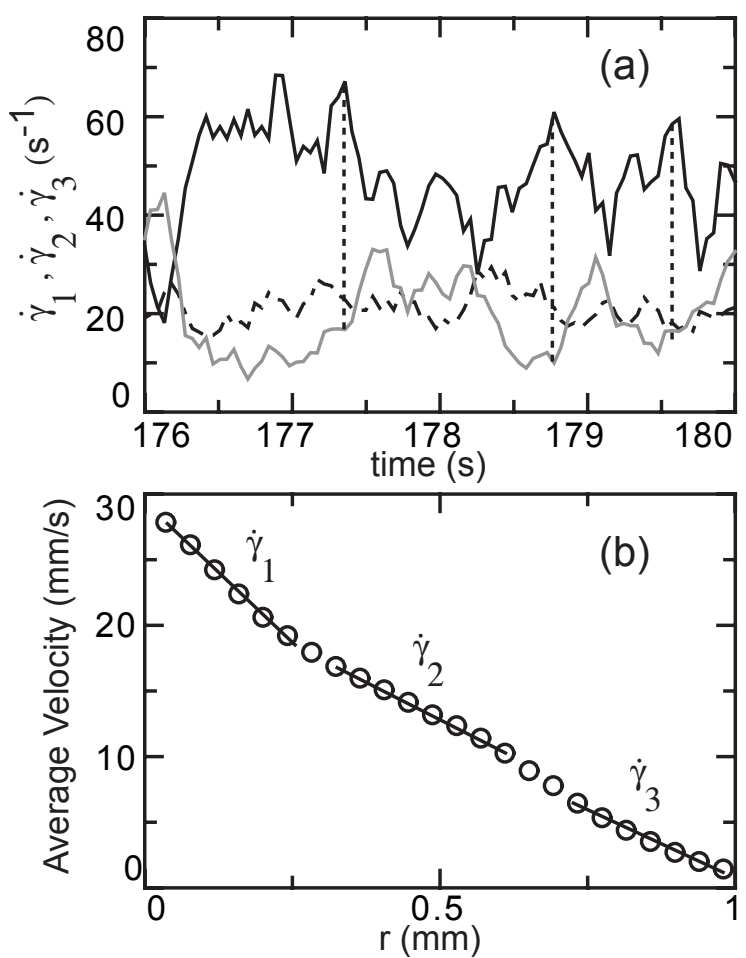

Fig. 9. (a) Local shear rates $\left(\dot{\gamma}_{1}, \dot{\gamma}_{2}, \dot{\gamma}_{3}\right)$ as a function of time for a shear stress of 17 Pa. The black line corresponds to the highly sheared band $\left(\dot{\gamma}_{1}\right)$ near the rotor, the dotted line to the shear band in the middle $\left(\dot{\gamma}_{2}\right)$ and the gray line corresponds to the weakly sheared band $\left(\dot{\gamma}_{3}\right)$ near the stator. (b) Time-averaged velocity profile as shown in Fig. $6 \mathrm{~b}$ indicating the regions used to fit the velocity profiles to obtain the local shear rates 
could be done by checking the profiles for any temporal evolution which would indicate if a transparent or turbid band is tested. It is known from previous investigations that the flip-flop like motion depends on gap size and applied shear stress $[25,26]$. For the Couette geometry used here, a temporal change of band structure within roughly half second is excepted. Indeed by analysing the oscillating shear rate signals (both, $\dot{\gamma}_{1}, \dot{\gamma}_{2}$ ) using FFT method we obtain an average frequency of $2 \mathrm{~Hz}$ or a period of $0.5 \mathrm{~s}$. This to some extent confirms the presence of alternating vorticity bands and unambiguously shows the coexistence of both radial and vorticity bands in the gap.

Decruppe et. al [8] have also observed similar oscillations in the velocity profiles measured in the cetyltrimethylammonium bromide (CTAB)-NaSal system in the narrow shear thickening region $6.5 \leqslant \dot{\gamma} \leqslant 7.5 \mathrm{~s}^{-1}$, at the end of the stress-plateau regime. They particularly observed a monotonous variation of the velocity in the gap along with very large fluctuations of the local velocity. Comparing our work to the system studied by Decruppe et.al, we probably believe that a similar situation (coexistence of radial and vorticity bands) may be existing in their systems as well. Analyzing our data thoroughly, we can however conclude that the velocity profiles in the shear-thickening regime do show a change in band shear rate with a period of half a second as expected. Although the velocity signal is extremely noisy and therefore frequency analysis is not as accurate as in the case of our previous work [25,26], we still postulate a banding situation as depicted in Fig. 1d where radial and vorticity bands coexist.

\subsection{Strain-controlled experiments}

In this section, we very briefly report on the local velocity measurements carried out in the strain-controlled mode of the rheometer. Similar to the experiments in the earlier section the measurements are carried out in the Newtonian, shear-thinning (stress-plateau) and shear-thickening flow regime. As shown in Fig. 10, the flow is homogenous in the Newtonian and shear-thinning flow regions $\left(\dot{\gamma} \leqslant 15 \mathrm{~s}^{-1}\right)$. However, the velocity profile is somewhat inhomogenous in the shear-thickening regime $\left(15 \mathrm{~s}^{-1} \leqslant \dot{\gamma} \leqslant 70 \mathrm{~s}^{-1}\right)$ as depicted in Fig. 11. But, the pronounced two-banded situation that was observed in stress-controlled experiments (Fig. 6) is not seen in shear rate controlled measurements. It seems that either the flow is almost homogenous or chaotic in the shear rate controlled mode. Moreover, in shear rate controlled experiments wall slip is sometimes detected at the rotating wall for a shear rate of $15 \mathrm{~s}^{-1}$ and such wall slip persists till about only $20 \mathrm{~s}^{-1}$. However, with our measurements in the Couette shear cell we did not observe a systematic wall slip and hence it is difficult to relate the slip phenomena to the observed shear banding flow.

\section{Summary}

In this study we have described and discussed local velocity measurements performed using ultrasould velocimetry on a equimolar $(40 \mathrm{mM})$ cetylpyridinium chloride-sodium salicylate mixture forming wormlike micelles. When the shear stress is increased the solution exhibits Newtonian, shear-thinning and shear-thickening behavior, respectively. In the shear-thickening regime, transparent and turbid bands appear in the vorticity direction of the Couette geometry. These vorticity bands alternate in their position (flip-flop), forcing the free parameter (shear rate, $\dot{\gamma}$, apparent viscosity, $\eta$ ) 

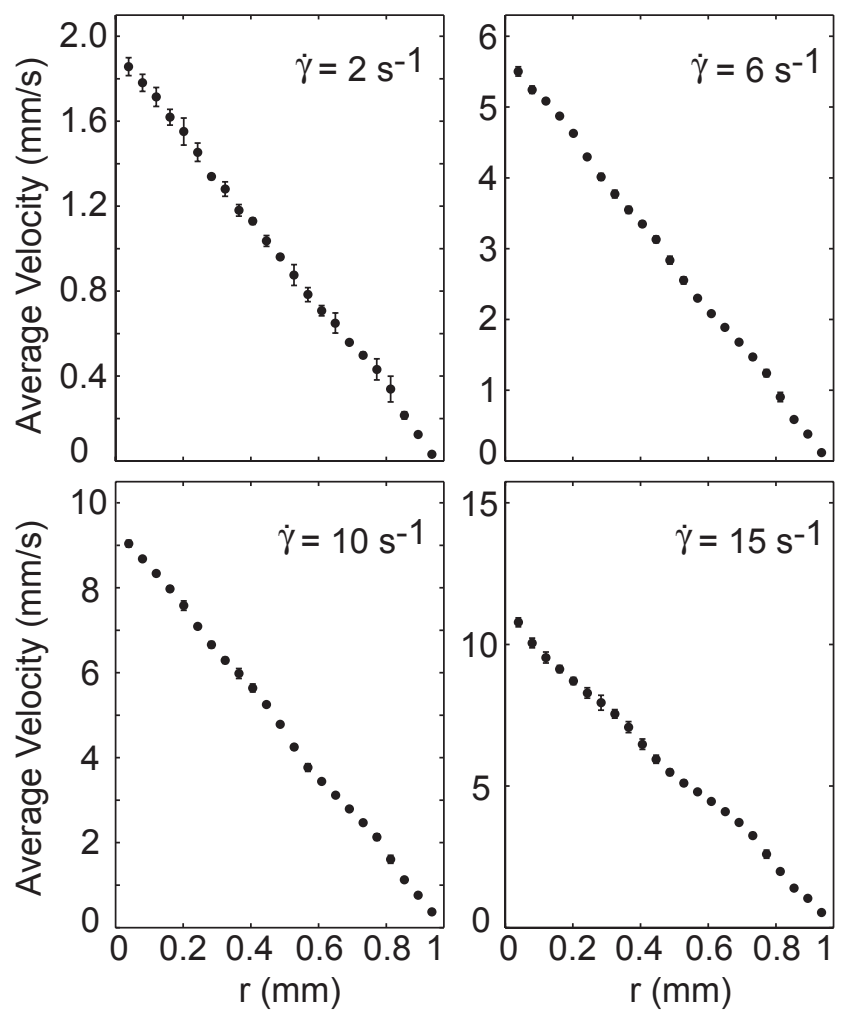

Fig. 10. Time-averaged velocity profiles for different shear rates in the Newtonian $\left(2\right.$ and $\left.6 \mathrm{~s}^{-1}\right)$ and shear-thinning $(10$ and $15 \mathrm{~s}^{-1}$ ) regimes measured in the strain-controlled mode. Error bars correspond to the standard deviation of the local velocities and mainly account for temporal fluctuations in the velocity. $r$ denotes the radial distance to the rotor; $r=0$ at the rotor and $r=1$ at the stator.

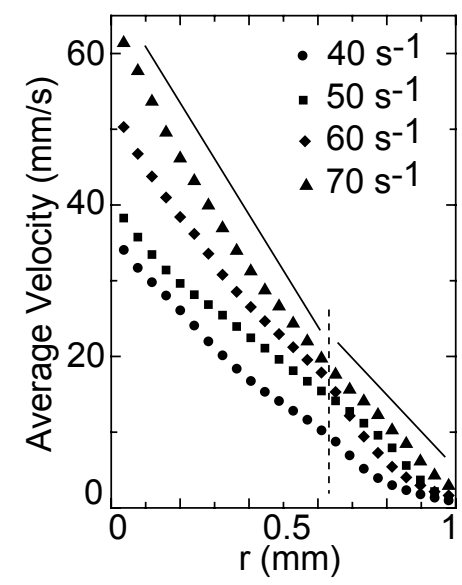

Fig. 11. Time-averaged velocity profiles for different shear rates in the shear-thickening regime measured in the strain-controlled mode. The dotted vertical line indicates the position of the interface between the radial bands and the solid lines are guides to the eye. $r$ denotes the radial distance to the rotor; $r=0$ at the rotor and $r=1$ at the stator.

to oscillate under controlled shear stress conditions. Point-wise local velocity measurements using high frequency ultrasonic velocimetry, revealed homogenous flow inside the gap of the Couette cell in the stress-plateau region. This is in sharp contrast to other wormlike micellar systems, where a two banded flows have been observed. However, when the solution is sheared beyond the critical shear stress (shear-thickening regime), a two-banded situation appears in the gap of the Couette cell showing the presence of a highly sheared band $\left(\dot{\gamma}_{1}\right)$ at the rotating wall and a weakly 
sheared band $\left(\dot{\gamma}_{2}\right)$ at the stationary wall. When further increasing the shear stress, the flow inside the gap becomes even more complex with the presence of more than two radial shear bands. To our knowledge this is the first report on the presence of radial shear bands in this $40 \mathrm{mM} \mathrm{CPyCl}-\mathrm{NaSal}$ wormlike micellar system.

With the help of very rapid data aquisition, fast UVP measurements were performed in the shear thickening regime to capture the interface dynamics and the temporal behavior of the local shear rates in the shear bands. Such measurements indicated a very complex time dependent flow behavior with large temporal oscillations in the velocity profile. The frequency of the oscillations in the velocity profiles corresponded to that of appearance and disappearance of vorticity bands $(0.5 \mathrm{~s})$. This gives an indication for the co-existance of both radial and vorticity banding in this system. Furthermore, the UVP measurements performed under shear rate controlled mode of the rheometer did not show significant radial shear banding behavior in the shear-thickening flow region. These experiments indicate that the flow behavior of shear-thickening $40 \mathrm{mM} \mathrm{CPyCl-NaSal} \mathrm{is} \mathrm{very} \mathrm{complex} \mathrm{with} \mathrm{the} \mathrm{presence} \mathrm{of} \mathrm{both} \mathrm{radial} \mathrm{and} \mathrm{vorticity}$ bands. We are currently in the process of investigating the presence of coexsisting vorticity and radial bands with a sub-structure in the radial bands by direct birefringence measurements. Our preliminary birefringence experiments show different local material stages within the radial bands under non-monotonic flow conditions. We will be presenting these results in a forthcoming article.

\section{References}

1. H. Rehage, H. Hoffmann, Mol. Phys. 74(5), 933 (1991)

2. C. Grand, J. Arrault, M.E. Cates, J. Phys. II 7(8), 1071 (1997)

3. T. Shikata, H. Hirata, T. Kotaka, Langmuir 3(6), 1081 (1987)

4. A.M. Wunderlich, P.O. Brunn, Colloid Polym. Sci. 267(7), 627 (1989)

5. P. Fischer, Rheol. Acta 39, 234 (2000)

6. E.K. Wheeler, P. Fischer, G.G. Fuller, J. Non-Newton. Fluid Mech. 75(2-3), 193 (1998)

7. B.M. Marin-Santibanez, J. Perez-Gonzalez, L. de Vargas, F. Rodriguez-Gonzalez, G. Huelsz, Langmuir 22(9), 4015 (2006)

8. J.P. Decruppe, O. Greffier, S. Manneville, S. Lerouge, Phys. Rev. E 73, 061509 (2006)

9. B.A. Schubert, N.J. Wagner, E.W. Kaler, S.R. Raghavan, Langmuir 20(9), 3564 (2004)

10. H. Azzouzi, J.P. Decruppe, S. Lerouge, Eur. Phys. J. E 17(4), 507 (2005)

11. J.F. Berret, D.C. Roux, G. Porte, P. Lindner, Europhys. Lett. 25(7), 521 (1994)

12. P. Boltenhagen, Y.T. Hu, E.F. Matthys, D.J. Pine, Phys. Rev. Lett. 79(12), 2359 (1997)

13. P. Fischer, E.K. Wheeler, G.G. Fuller, Rheol. Acta 41, 35 (2002)

14. I.A. Kadoma, J.W. van Egmond, Phys. Rev. Lett. 80(25), 5679 (1998)

15. R. Oda, P. Panizza, M. Schmutz, F. Lequeux, Langmuir 13(24), 6407 (1997)

16. N.A. Spenley, X.F. Yuan, M.E. Cates, J. Phys. II France 6(4), 551 (1996)

17. P.D. Olmsted, C.Y.D. Lu, Phys. Rev. E 60(4), 4397 (1999)

18. S.M. Fielding, P.D. Olmsted, Eur. Phys. J. E 11(1), 65 (2003)

19. V. Schmitt, C.M. Marques, F. Lequeux, Phys. Rev. E 52(4), 4009 (1995) 
20. J.P. Decruppe, R. Cressely, R. Makhloufi, E. Cappelaere, Colloid Polym. Sci. 273(4), 346 (1995)

21. S. Lerouge, J.P. Decruppe, C. Humbert, Phys. Rev. Lett. 81(24), 5457 (1998)

22. E. Fischer, P.T. Callaghan, Phys. Rev. E 64(1), 011501 (2001)

23. L. Becu, S. Manneville, A. Colin, Phys. Rev. Lett. 93(1), 018301 (2004)

24. S. Manneville, J.B. Salmon, L. Becu, A. Colin, F. Molino, Rheol. Acta 43(5), 408 (2004)

25. V. Herle, P. Fischer, E.J. Windhab, Langmuir 21(20), 9051 (2005)

26. V. Herle, J. Kohlbrecher, B. Pfister, P. Fischer, E.J. Windhab, Phys. Rev. Lett. p. accepted for publication (2007)

27. P. Hu, Y. T. Boltenhagen, D.J. Pine, J. Rheol. 42(5), 1185 (1998)

28. L. Hilliou, D. Vlassopoulos, Ind. Eng. Chem. Res. 41(25), 6246 (2002)

29. S.M. Fielding, P.D. Olmsted, Phys. Rev. Lett. 92(8), 084502 (2004)

30. R. Ganapathy, A.K. Sood, Phys. Rev. Lett. 96(10), 108301 (2006)

31. M.E. Cates, S.M. Fielding, Adv. Phys. 55(7-8), 799 (2006)

32. J.L. Goveas, D.J. Pine, Europhys. Lett. 48(6), 706 (1999)

33. M.E. Cates, S.J. Candau, Europhys. Lett. 55(6), 887 (2001)

34. A. Aradian, M.E. Cates, Europhys. Lett. 70(3), 397 (2005)

35. S. Manneville, L. Becu, A. Colin, Eur. Phys. J.-Appl. Phys 28(3), 361 (2004)

36. J.F. Berret, Langmuir 13(8), 2227 (1997)

37. J.F. Berret, G. Porte, J.P. Decruppe, Phys. Rev. E 55(2), 1668 (1997)

38. J.I. Escalante, E.R. Macias, F. Bautista, J.H. Perez-Lopez, J.F.A. Soltero, J.E. Puig, O. Manero, Langmuir 19(17), 6620 (2003)

39. J.P. Decruppe, S. Lerouge, J.F. Berret, Phys. Rev. E 63(2), 022501 (2001)

40. N.A. Spenley, M.E. Cates, T.C.B. McLeish, Phys. Rev. Lett. 71(6), 939 (1993)

41. F. Bautista, J.F.A. Soltero, E.R. Macias, J.E. Puig, O. Manero, J. Phys. Chem. B 106(50), 13018 (2002)

42. J.F. Berret, D.C. Roux, G. Porte, J. Phys. II 4(8), 1261 (1994)

43. J.F. Berret, R. Gamez-Corrales, J. Oberdisse, L.M. Walker, P. Lindner, Europhys. Lett. 41(6), 677 (1998)

44. J.B. Salmon, S. Manneville, A. Colin, Phys. Rev. E 68(5), 051503 (2003)

45. S. Manneville, J.B. Salmon, A. Colin, Eur. Phys. J. E 13(2), 197 (2004)

46. J.B. Salmon, A. Colin, D. Roux, Phys. Rev. E 66(3), 031505 (2002)

47. E. Cappelaere, J.F. Berret, J.P. Decruppe, R. Cressely, P. Lindner, Phys. Rev. E 56(2), 1869 (1997)

48. A.F. Mendez-Sanchez, M.R. Lopez-Gonzalez, V.H. Rolon-Garrido, J. Perez-Gonzalez, L. de Vargas, Rheol. Acta 42(1-2), $56(2003)$

49. A.F. Mendez-Sanchez, J. Perez-Gonzalez, L. de Vargas, J.R. Castrejon-Pita, A.A. Castrejon-Pita, G. Huelsz, J. Rheol. $\mathbf{4 7}(6), 1455(2003)$

50. M.M. Britton, R.W. Mair, R.K. Lambert, P.T. Callaghan, J. Rheol. 43(4), 897 (1999)

51. G. Picard, A. Ajdari, L. Bocquet, F. Lequeux, Phys. Rev. E 66(5), 051501 (2002)

52. J.K.G. Dhont, Phys. Rev. E 60(4), 4534 (1999)

53. P.D. Olmsted, P. Goldbart, Phys. Rev. A 41(8), 4578 (1990)

54. E. Fischer, P.T. Callaghan, Europhys. Lett. 50(6), 803 (2000) 